Proc. of the 13th Symposium of Magnetic Measurements and Modeling SMMM'2018, Wieliczka, Poland, October 8-10, 2018

\title{
Predicting Recoil Curves in Stoner-Wohlfarth Anisotropic Magnets
}

\author{
M.F. DE CAMpos* And J.A. DE CASTro \\ Universidade Federal Fluminense, Volta Redonda 27255-125, Brazil
}

\begin{abstract}
It is possible to predict recoil curves in magnetic materials following the Stoner-Wohlfarth behavior. In the presented example, the shapes of recoil curves are predicted for anisotropic Stoner-Wohlfarth magnets, which follow distribution of type $f=\cos ^{n}(\alpha)$, with $n=15$. As the predicted recoil curves are near the experimentally observed ones for SmCoCuFeZr magnets, this implies that the coercivity mechanism is coherent rotation for these magnets.
\end{abstract}

DOI: 10.12693/APhysPolA.136.737

PACS/topics: 75.50.Vv, 75.50.Ww, 75.60.Jk, 75.60.-d

\section{Introduction}

The Stoner-Wohlfarth (SW) model [1] has several assumptions, among them phases with uniaxial easy axis and non-interacting particles. A very important assumption is monodomain particles. High coercivity $\mathrm{Sm}_{2} \mathrm{Co}_{17}$ type magnets (5 element alloy $\mathrm{Sm}-\mathrm{Co}-\mathrm{Cu}-\mathrm{Fe}-\mathrm{Zr}$ ) have shown behavior close to the $\mathrm{SW}$ prediction $[2,3]$. Here, it is briefly discussed how to predict recoil curves for anisotropic Stoner-Wohlfarth magnets $[4,5]$. This is direct consequence of the SW model, where the initial magnetization curve can be predicted as the average between the 1st and 4th quadrants of the hysteresis.

In hard magnetic materials manufactured by powder metallurgy, as $\mathrm{SmCo}_{5}[6,7], \quad \mathrm{NdFeB}[8]$, or $\mathrm{SmCoFeCuZr}[2]$, the particles are milled until they are a single crystal. Then there is a step of alignment of crystals under magnetic field. After the pressing step, the sample is sintered and heat treated. This processing introduces a strong uniaxial texture, which can be described by a symmetrical distribution, as a Gaussian, a Lorentzian, or $f=\cos ^{n}(\alpha)[4,5]$. The function $f=$ $\cos ^{n}(\alpha)$ gives a very similar result when compared with a Gaussian for high $n$ values ( $n$ above 10). Thus, a symmetrical distribution describes the orientation of the crystals (or grains) in the space, also called crystallographic texture. For higher $n$, the alignment of the particles is better. The ideal situation is when $n \rightarrow \infty$.

\section{The Stoner-Wohlfarth model}

The SW model considers only two energy terms: one due to external field $H$, and another due to magnetocrystalline anisotropy $K_{1}$. The angle between external field and crystal easy axis is $\alpha$. The angle between $M_{s}$ and the easy axis is $\theta . M_{s}$ is the magnetization in saturation.

\footnotetext{
*corresponding author; e-mail: marcosflavio@id.uff.br
}

$M_{r}$ is remanence. By making $\mathrm{d} E / \mathrm{d} \theta=0$ and $\mathrm{d}^{2} E / \mathrm{d} \theta^{2}=0$, the critical field $h_{c}$ for irreversible rotation is obtained. It is typical to use the reduced quantities $m$ and $h$, where $m=M / M_{s}$ and $h=H / H_{A} . H_{A}$ is the anisotropy field, given by $2 K_{1} / M_{s}$.

$$
E=-H M_{S} \cos (\theta-\alpha)+K_{1} \sin ^{2} \theta .
$$

The SW model assumes uniaxial anisotropy, i.e., only one easy axis. In the Stoner-Wohlfarth model, there are two points of minimum: magnetized in one direction and in another direction, see Fig. 1. For one crystal perfectly aligned in relation to the magnetic field, a perfect square hysteresis is obtained, as shown in Fig. 1.

The critical field $h_{c}$ for irreversible rotation as function of grain orientation is given by Eq. (2), with $t$ given by Eq. (3). As shown in Fig. $2, h_{c}$ is between 0.5 and 1.0. As $h_{c}$ gives the field where irreversiblerotation

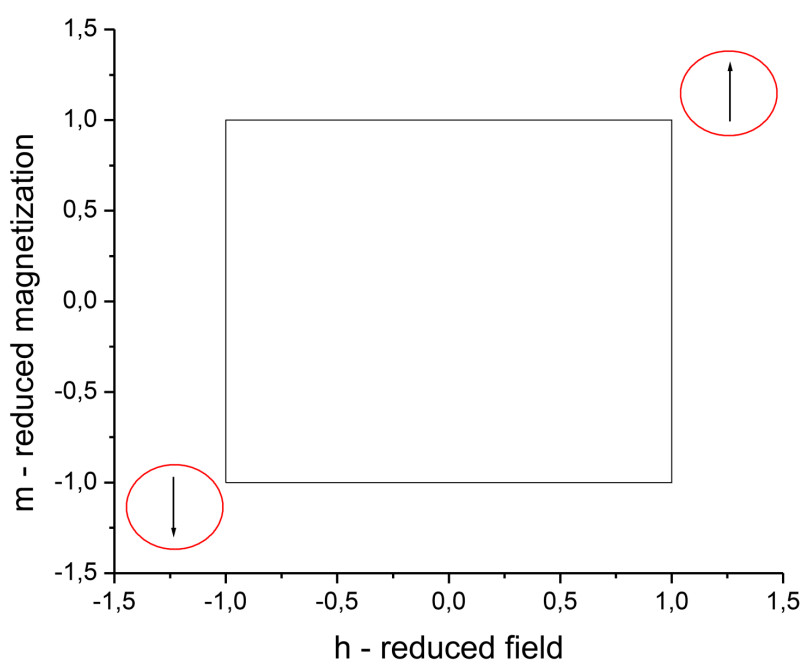

Fig. 1. Hysteresis curve for a grain with perfect alignment, i.e., $\alpha=0$. The Stoner-Wohlfarth model considers only two minimums of energy: aligned in one direction and aligned in an opposite direction. 


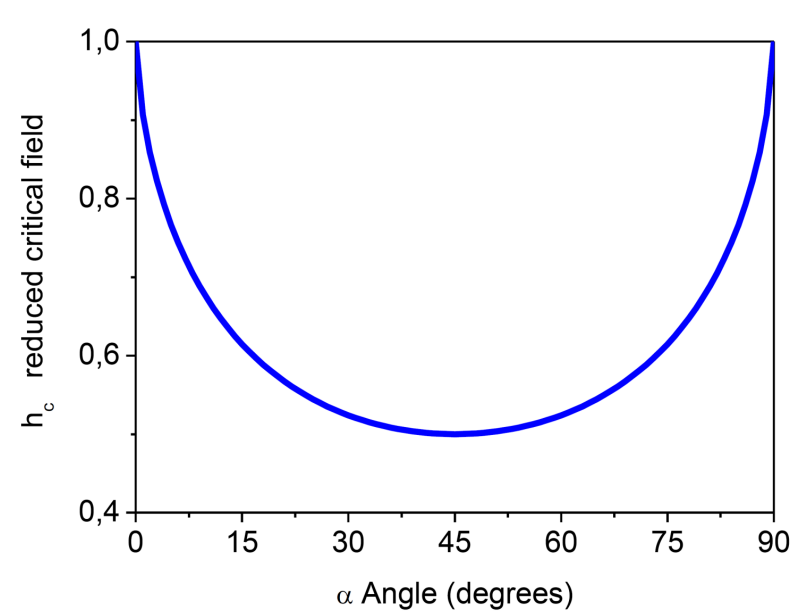

Fig. 2. Reduced field $h_{c}$ as function of an angle $\alpha$. According to the SW model, there are regions of reversible and irreversible rotation. For $-0.5 \leq h_{c} \leq 0.5$, always there is reversible rotation.

takes place, then in the region, where $-0.5<h_{c}<0.5$ only reversible rotation takes place, without inversion of the magnetization vector.

$$
\begin{aligned}
& h_{c}=\frac{\left(1-t^{2}+t^{4}\right)^{1 / 2}}{1+t^{2}} \\
& t=(\tan \alpha)^{1 / 3}
\end{aligned}
$$

\section{Model for recoil curves of hysteresis}

The SW model can be easily extended for the anisotropic case, by means of a symmetrical function, for example a Gaussian or a Lorentzian [5]. As mentioned before, the $f=\cos ^{n}(\alpha)$ distribution gives a result near the Gaussian distribution for well aligned magnets, with $M_{r} / M_{s}$ above 0.9 [5]. In the demagnetized condition half of the grains are magnetized in one direction and another half in the another direction. For an isotropic SW magnet, the initial magnetization curve is given by Fig. 3. For a magnet with perfect alignment, the initial magnetization curve (see the curve in blue) is presented in Fig. 4. In other words, in the SW model, the initial magnetization curves is the average between the 1st and 4th quadrant of the hysteresis [1]. An anisotropic hysteresis curve for $n=15$ was calculated as can be seen in Fig. 5, which also shows the initial magnetization curve. It should be again reminded, as can be seen in Fig. 1, that there are only two minimums of energy, with the crystals magnetized either in one direction or in the opposite direction. Thus, inside the region of reversible rotation, the same percentage of grains are magnetized either in one direction or the opposite.

From Fig. 2, it is observed that irreversible rotation only takes place for $h_{c}>0.5$ or $h_{c}<-0.5$, see Fig. 6 . In the same way as it is possible to predict initial

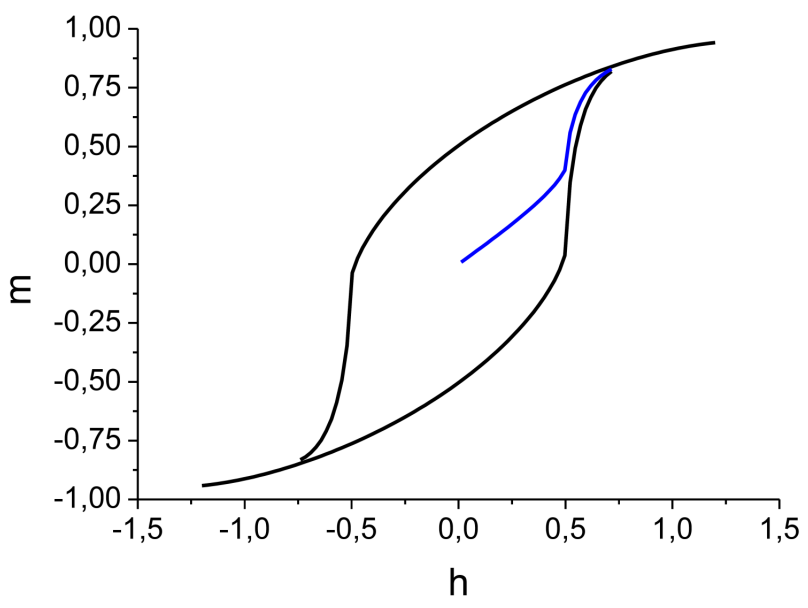

Fig. 3. Initial magnetization curve (blue curve) for an isotropic Stoner-Wohlfarth magnet, i.e. $n=0$ in a distribution of the type $f=\cos ^{n}(\alpha)$.

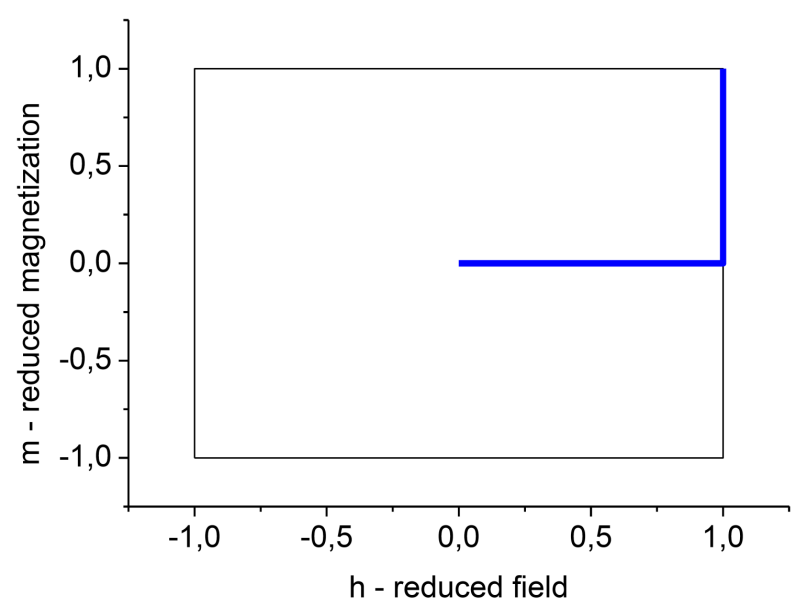

Fig. 4. Initial magnetization curve (blue curve) for a Stoner-Wohlfarth magnet with perfect alignment, i.e., $n \rightarrow \infty$ in a distribution of the type $f=\cos ^{n}(\alpha)$.

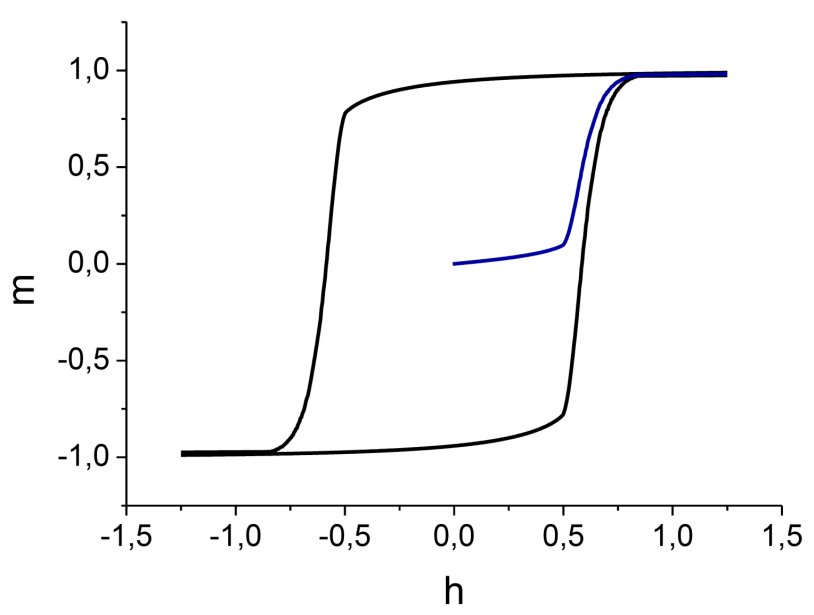

Fig. 5. Initial magnetization curve for a $f=\cos ^{n}(\alpha)$ distribution with $n=15$. 


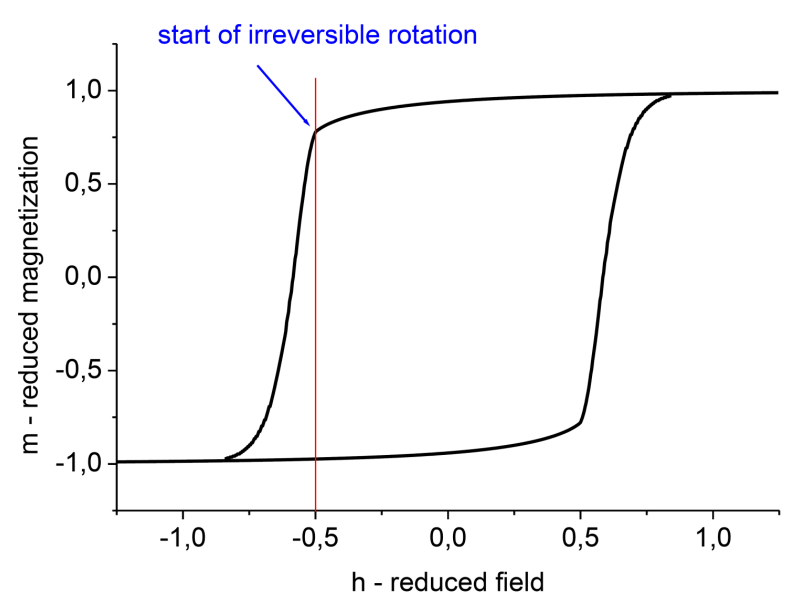

Fig. 6. Start of irreversible rotation for an anisotropic Stoner-Wohlfarth magnet, which follows distribution $f=\cos ^{n}(\alpha)$, with $n=15 . \quad M_{r} / M_{s}=n+1 / n+2$, then $M_{r} / M_{s}=0.94$ for this magnet.

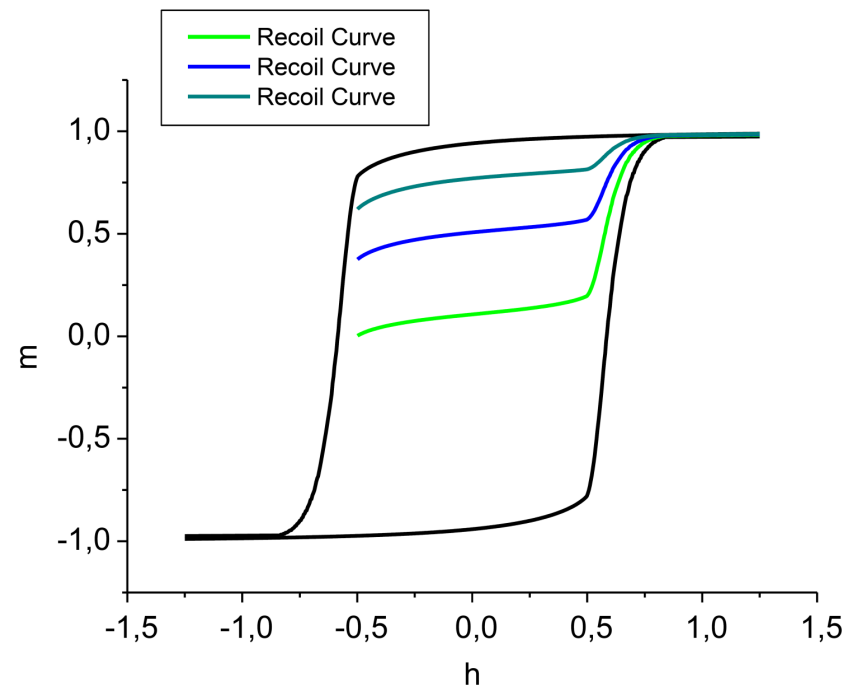

Fig. 7. Several possible recoil curves. It is assumed an anisotropic Stoner-Wohlfarth magnet, which follows distribution of orientation $f=\cos ^{n}(\alpha)$, with $n=15$.

magnetization curves, then it is also possible the prediction of recoil curves, as shown in Fig. 7. As the predicted recoil curves are near the experimentally observed for 2:17 type SmCo magnets [9], this implies that the coercivity mechanism is coherent rotation for these magnets, as already noted before $[2,3]$.

Deviations from the SW model usually are due to interaction between particles. In the high coercivity SmCo 2:17 magnets, there is formation of a copper-rich paramagnetic $\mathrm{Sm}(\mathrm{Cu}, \mathrm{Co})_{5}$ cell phase surrounding the $\mathrm{Sm}_{2}(\mathrm{CoFe})_{17}$ nanocrystalline grains [3]. The copper-rich cell avoids interaction between the particles, and a behavior near that predicted by the SW model can be observed [2,3]. The low susceptibility (or low permeability) of the initial magnetization curve in thermally demagnetized samples, as shown in Figs. 4 and 5, is clear indication of coherent rotation (the Stoner-Wohlfarth mode) as coercivity mechanism.

\section{Conclusions}

Recoil curves were calculated for magnetic materials by applying the Stoner-Wohlfarth model supplemented by a symmetrical distribution $f=\cos ^{n}(\alpha)$. The origin of the shape of the recoil curves are predicted for anisotropic $\mathrm{SW}$ magnets. As the predicted recoil curves are near the experimentally observed ones for SmCo 2:17 type magnets magnets, this again confirms coherent rotation as coercivity mechanism for these magnets.

\section{Acknowledgments}

Work supported in part by FAPERJ (Fundação Carlos Chagas Filho de Amparo à Pesquisa do Estado do Rio de Janeiro) and $\mathrm{CNPq}$ (Conselho Nacional de Desenvolvimento Científico e Tecnológico).

\section{References}

[1] E.C. Stoner, E.P. Wohlfarth, IEEE Trans. Magn. 27, 3475 (1991).

[2] F.A. Sampaio da Silva, N.A. Castro, M.F. de Campos , J. Magn. Magn. Mater. 328, 53 (2013).

[3] S.A. Romero, M.F. de Campos, J.A. de Castro, A.J. Moreira, F.J.G. Landgraf, J. Alloys Comp. 551, 312 (2013).

[4] M.F.D. Campos, F.A.S. Silva, J.A. de Castro, Mater. Sci. Forum 775, 431 (2014).

[5] M.F.d. Campos, F.A.S. da Silva, E.A. Perigo, J.A. de Castro, J. Magn. Magn. Mater. 345, 147 (2013).

[6] M.F.D. Campos, T. Yonamine, M. Fukuhara, R. Machado, S.A. Romero, F.J.G. Landgraf, D. Rodrigues, F.P. Missell, J. Appl. Phys. 101, 09K516 (2007).

[7] M.F.D. Campos, F.J.G. Landgraf, R. Machado, D. Rodrigues, S.A. Romero, A.C. Neiva, F.P. Missell, J. Alloys Comp. 267, 257 (1998).

[8] C.G. Hauegen, F.R.F da Silva, F.A. Sampaio da Silva, J.F.C Lins, M.F. de Campos, Mater. Sci. Forum 727, 135 (2012).

[9] G. Bavendiek, S. Steentjes, J. Sabirov, K. Hameyer, in: Proc. of WMM'18, Dresden (Germany), 2018, p. 148. 